\title{
The Incorporation of fixed cost and multilevel capacities into the discrete and continuous single source capacitated facility location problem
}

\author{
Chandra Ade Irawan • Martino Luis • Said \\ Salhi · Arif Imran
}

Received: date / Accepted: date

\begin{abstract}
In this study we investigate the single source location problem with the presence of several possible capacities and the opening (fixed) cost of a facility that is depended on the capacity used and the area where the facility is located. Mathematical models of the problem for both the discrete and the continuous cases using the Rectilinear and Euclidean distances are produced. Our aim is to find the optimal number of open facilities, their corresponding locations, and their respective capacities alongside the assignment of the customers to the open facilities in order to minimise the total fixed and transportation cost. For relatively large problems, two solution methods are proposed namely an iterative matheuristic approach and VNS-based matheuristic technique. Dataset from the literature is adapted to assess our proposed methods. To assess the performance of the proposed solution methods, the exact method is first applied to small size instances where optimal solutions can be identified or lower and upper bounds can be recorded. Results obtained by the proposed solution methods are also reported for the larger instances.
\end{abstract}

Keywords Discrete and continuous capacitated location - Opening fixed cost · Mathematical formulation · Matheuristic · VNS

Chandra Ade Irawan

Nottingham University Business School China, University of Nottingham Ningbo China, 199 Taikang East Road, Ningbo 315100, China

E-mail: chandra.irawan@nottingham.edu.cn

Martino Luis

College of Engineering, Mathematics, and Physical Sciences, University of Exeter, Exeter EX4 $4 \mathrm{QF}, \mathrm{UK}$

E-mail:

Said Salhi

Centre for Logistics \& Heuristic Optimization (CLHO), Kent Business School, University of Kent, Canterbury, CT2 7PE, UK

E-mail: S.Salhi@kent.ac.uk

Arif Imran

Department of Industrial Engineering, Institut Teknologi Nasional, Bandung 40124, Indonesia E-mail: arifimr@yahoo.com 


\section{Introduction}

Facility location problem arises whenever there is the question of locating one or more new facilities with respect to a set of existing facilities (discrete case) or on the plane (continuous case) with the aim to optimise one or more criteria (Drezner and Hamacher, 2002; Hale and Moberg, 2003; Drezner et al, 2006; Daskin, 2008; Brimberg et al, 2008; Farahani et al, 2014). The location problems may be classified based on topography, capacity restrictions, or sourcing types. Based on the topography of the site selection, location problems can be divided into continuous and discrete models. In the continuous location models, the facilities can be located anywhere on the plane (i.e. there is an infinite number of possible sites) whereas in the discrete case, there is a finite number of potential sites from which to select the new facilities. In terms of supplying abilities of the facilities, the problem may be classified as uncapacitated or capacitated. In the uncapacitated case, the demand at each customer location is supplied by the nearest facility at a minimum cost (see Gamal and Salhi 2001 and 2003). This is not true when the facilities have capacity restrictions as some customers may need to be served by farther facilities given their nearest facilities may not be large enough to accommodate all their entire demands. The allocation problem in this situation is found by solving a transportation or an assignment problem instead.

The location problems may further be categorized by a sourcing type which considers multiple or single sourcing. The former is known as the multi-source type-problem where customer demands can be served by more than one facility whereas in the latter, the problem is referred to as a single source type-problem when a customer demand is satisfied only from one facility. The single source constraint is known to be much harder to tackle compared to its counterpart (Klose and Drexl, 2005) due to solving the assignment problem instead of the transportation problem. In this paper, we investigate both continuous and discrete location problems with single sourcing. In the discrete case, this study focuses on a variant of facility location problem by including capacity restrictions known as the Capacitated Facility Location Problem (CFLP). Here, we are given a set of potential facility locations with their fixed costs as well as a set of customers with known demands. The aim is to find the number of facilities to open their locations, their respective capacities and to find the assignment of these customers to these open facilities without violating the capacity of any of the open facilities. The objective is to minimise the sum of the total fixed and the transportation costs.

In the continuous case, this study relates to the well-known Capacitated Multifacility Weber problem (CMFWP) where the problem entails to find the locations of $\mathrm{M}$ facilities in the plane and their customer allocations without violating the capacity of any of the facilities to minimise the sum of opening and transportation costs. The CMFWP is considered NP-hard as shown by Megiddo and Supowit (1984) and Sherali and Nordai (1988). Most of the research in the CMFWP uses a constant capacity while incorporating the iterative Alternate Location-Allocation (ALA) heuristic of Cooper into the search (see for instance, Zainuddin and Salhi 2007; Luis et al 2009 and 2011, Manzour-al Ajdad et al 2012; Manzour et al 2013; Öncan 2013). In practice, it is also worth considering the presence of facility fixed/opening costs. Fixed cost can be defined as the set up or opening cost of a facility where it may be dependent on geographical (location) areas and/or a throughput rate (capacity) of the facility. For instance, governments apply different 
tax policies for urban, suburban, and remote regions or regional restrictions as some areas are under government protections such as forests, lakes, rivers, etc. In other words, some areas may have cheaper costs of establishing a facility whereas others may have very high costs. In this paper we incorporate the fixed cost that is based on several possible Capacities. We refer to this problem as the Single Source Facility Location Problem with fixed costs (SSFLPFC).

i. to propose mathematical models of the SSFLPFC problem for both the discrete and the continuous cases using the Rectilinear and Euclidean distances.

ii. to produce a linearisation scheme for the continuous case.

iii. to design two solution methods based on matheuristic approach for tackling relatively large SSFLPFC instances.

iv. to report new best solutions for benchmarking purposes.

The paper is structured as follows. A review of the relevant literature is given in the next section. The mathematical models of the SSFLPFC for both the discrete and the continuous cases are presented in Section 3 which also includes the linearisation scheme for the continuous case. Section 4 presents a detailed description of the proposed matheuristic to tackle both cases and Section 5 is devoted to the computational results. Finally, in Section 6, conclusions and some research avenues are highlighted.

\section{Literature Review}

This section presents works which are closely relevant to our problems for both the discrete case and the continuous case.

\subsection{Discrete capacitated facility location problem}

The capacitated facility location problem (CFLP) has received much attention in the literature. Here, we concentrate our review on the class of single source, known as the Single Source Capacitated Facility Location Problem (SSCFLP) by focussing on the last decade only. The interested reader may refer to Eiselt and Marianov (2011), Rahmani and MirHassani (2014), and Farahani et al (2014) for a comprehensive review.

Arostegui Jr. et al (2008) compare the performances of tabu search, simulated annealing, and genetic algorithm to solve facility location problems. The authors implement the three metaheuristics to solve the CFLP, the multi-period FLP, and the multi-commodity FLP. Their findings show that tabu search is the best performer in most cases. Contreras and Díaz (2008) present a scatter search heuristic method by embedding GRASP and tabu search to construct the improvement phase. Chen and Ting (2008) tackle the SSCFLP by designing a hybrid heuristic based on Lagrangian heuristic and ant colony system. Yang et al (2012) put forward an exact method based on a cut-and-solve scheme to deal with the SSCFLP. Guastaroba and Speranza (2014) enhance the Kernel Search heuristic to general Binary Integer Linear Programming problems to solve the SSCFLP. Ho (2015) studies the SSCFLP by proposing an iterated tabu search which uses randomized 
neighbourhood sampling and random perturbation mechanisms to diversify the search. For an overview and critical analysis on heuristic search, see Salhi (2017).

Several extensions on the CFLP are the work of Lin (2009) who investigates an extension of the single-source capacitated facility location problem by considering uncertain demand while requiring a specific service level. A hybrid heuristic based on lagrangean relaxation with a multi-exchange heuristic within a branchand-bound framework is put forward to deal with the problem. Aydin and Murat (2013) address the CFLP by considering the unreliability of facilities. A hybrid algorithm that uses particle swarm optimization and the sample average approximation technique is developed to tackle the problem. Gadegaard et al (2016) study bi-objective discrete facility location problems with a cost objective and a bottleneck objective where the model is applied to solve the CFLP.

\section{The multi-type capacitated facility location problem}

This section focuses on a variant of the capacitated facility location problems with several possible capacities which has close similarities to our problem. This problem is referred to the multi-type or modular capacitated facility location problem. Among the recent works include the work by Correia and Captivo (2003) who extended the problem to the case of single sourcing problem. Here, a Lagrangean heuristic is used to generate feasible solutions and then a two-phase heuristic procedure based on a greedy algorithm and tabu search is put forward to improve the solutions. Correia et al (2010a) design a discretization reformulation technique to tackle the CFLP with modular capacity levels and distribution costs. Two models are developed and solved as a simple knapsack problem. On the other hand, Correia et al (2010b) address the single-allocation hub location problems with multiple capacity levels. The problems are formulated as mixed-integer linear programming formulations.

\subsection{Continuous capacitated facility location problem}

Our literature review on the continuous case is divided into three parts. First, we look at the capacitated multi-facility Weber problem without fixed costs and then at the MFWP with the presence of fixed costs. Lastly, we discuss several works on the single-source capacitated multi-facility Weber Problem. We do not look at the MFWP, but the reader can refer to the recent papers of Brimberg et al (2008) and Brimberg et al (2014) for a comprehensive review.

\section{(i) the capacitated $M F W P(C M F W P)$}

The earliest work of CMFWP was proposed by Cooper (1972) who designs exact and heuristic methods. In the heuristic approach, the well-known alternating transportation-location (ATL for short) heuristic is put forward. ATL is basically a revision of the heuristic (ALA) originally developed by Cooper (1964) for the uncapacitated case. The idea behind this approach is to solve alternately the location-allocation problem and the Transportation Problem (TP) until there is no significant improvement found in the total cost. Among the recent works 
include Zainuddin and Salhi (2007) who investigate the Euclidean CMFWP by proposing a perturbation-based heuristic which considers borderline customers whose locations lie approximately half-way between their nearest and their second nearest facilities. Aras et al (2007a) propose three heuristic methods which include Lagrangean heuristic, the discrete $p$-capacitated facility location heuristic which is adopted from the $p$-median method of Hansen et al (1998), and the cellular heuristic of Gamal and Salhi (2003) to tackle the CMFWP with Euclidean, squared Euclidean, and $\ell_{p}$ distances. Aras et al (2007b) solve the same problem but with rectilinear, Euclidean, squared Euclidean, and $\ell_{p}$ distances by using simulated annealing, threshold accepting and genetic algorithms. In a subsequent study, Aras et al (2008) implement their earlier methods to deal with the problem with rectilinear distance. Luis et al (2009) design the concept of region-rejection into their constructive heuristic whereas Mohammadi et al (2010) propose two genetic algorithms (GAs); one for the location problem and the other for the allocation of customers to those open facilities. Luis et al (2011) introduce a novel guided reactive greedy randomised adaptive search procedure (GRASP) by embedding a framework that combines adaptive learning with the concept of restricted regions. Akyüs et al (2014) put forward two types of branch and bound algorithms where the first is based on the allocation space whereas the second on the partition of the location space.

\section{(ii) the SSCMFWP}

The investigation on the SSCMFWP lacks attention in the literature. Gong et al (1997) is one of the pioneers to study this problem by developing a hybrid evolutionary method based on a genetic algorithm to search the locatable area and hence find the global or near global locations. In the allocation stage, a Lagrangean relaxation approach is then applied. Fifteen years later, Manzour-al Ajdad et al (2012) tackle the SSCMFWP using an interesting iterative two phase heuristic algorithm. In the first phase, the location problem is solved by the ALA method of Cooper (1964) using two assignment rules namely the simplified and parallel assignments respectively. The second is the allocation stage where customers are allocated to facilities by solving the generalised assignment problem optimally. A heuristic approach based on simulated annealing is proposed as an alternative method to the first phase. Manzour et al (2013) also design a simpler version to the one proposed by Manzour-al Ajdad et al (2012) but with slightly inferior results. Öncan (2013) put forward three schemes to deal with the problem with Euclidean and Rectilinear distances. The first one is the Single-Source ALA method which is an enhanced version of Coopers ALA method (Cooper, 1964) when the allocation phase is solved optimally. The second one is a very large neighbourhood search heuristic which is embedded in the first method to allocate customers efficiently. In the third approach, a discrete approximation technique that uses a Lagrangean Relaxation procedure is put forward to find lower and upper bounding procedures. Competitive results were obtained when compared to the recent published work though these are found to be relatively inferior in some instances to those given by Manzour-al Ajdad et al (2012). 
(iii) the Weber problem with the presence of fixed cost

The literature on the facility location problems with fixed costs mainly concentrates on discrete location cases, see for instance, the works of Rahmani and MirHassani (2014), Guastaroba and Speranza (2014), Farahani et al (2014), and Ho (2015), whereas in the continuous location problem there is a lack of references. Brimberg et al. (2004) design a multiphase heuristic to tackle the MFWP with constant fixed costs. Initially, the problem is discretized by solving the simple plant location problem to get a good approximation of the number of facilities needed. Coopers location-allocation method is then applied to find the new improved locations of the facilities. A local search is carried out to observe whether having a few more or a few less facilities may give a better solution, as the fixed cost of adding/removing a few facilities is balanced by the decreased/increased transportation cost. Brimberg and Salhi (2005) study the Weber problem with single facility by proposing fixed costs that are zone-dependent, where zones are nonoverlapping convex polygons. A simple but powerful approach is put forward to optimally solve the problem. In the case of the multi facility problem, a discretization approach is also proposed. Both studies assume that there is no capacity restriction.

Luis et al (2015) apply the concept of restricted regions and a GRASP metaheuristic to solve the CMFWP with facility fixed costs. The authors present three types of fixed costs namely constant, zone-based, and continuous fixed cost functions. The four well-known data sets for the uncapacitated case from the literature are adapted to assess the performance of the proposed method with promising results.Hosseininezhad et al (2015) put forward a cross entropy heuristic to deal with CMFWP with zone-based fixed cost which considers production and installation costs. The experiments show that the proposed heuristic produces reasonably good results when compared to a GAMS optimizer. Very recently, Irawan et al (2017) propose a new model of the CMWFWP in the presence of facility fixed cost which is defined as capacity-based and zone-dependent where the number of facilities to be located is known in advance. A Heuristic solution framework based on the concept of restricted regions, a new priority-based assignment, and Coopers alternate location-allocation method is designed to tackle the problem. A metaheuristic approach that uses Variable Neighbourhood Search is also put forward. In addition, the authors construct a new dataset based on convex polygons using diamond and hexagonal shapes, some of these will be used in our study.

\section{The location models considering opening facility costs}

In this section, we present mathematical models of the location problem taking into account the cost of opening a facility. The opening cost comprises two types of cost which are based on the capacity and the location. The capacity-related cost is linked to the capacity used by the opening facility where higher capacity size may have a higher opening facility cost. The location cost depends on the area where the facility is established as some areas (especially in the big cities) could be more expensive than others. Mathematical models for the discrete case are first presented which are then followed by the ones for the continuous case. For both cases, two mathematical models are presented where the first one considers the 
capacity cost only while the second one also caters for the effect of the site in the selection of the facilities. For the discrete case, the location cost is based on the location of the potential facilities while for the continuous case, the cost is defined according to the area/zone where the open facility is sited. For the case of the continuous space, the proposed models could be considered as an enhancement of those given by Irawan et al (2017) where the number of open facilities is known in advance whereas here this number is a decision variable.

\subsection{Discrete location problems}

Two models are proposed namely the Discrete Location Problem with Capacity Cost (DLC-CC), and the Discrete Location Problem with both Capacity and Location Costs (DLP-CLC).

\section{A. The DLP-CC model}

In this the discrete location problem, the capacity cost is considered. The following notations are used to describe the sets, parameters, and decision variables.

\section{Sets and Parameters}

$I$ : set of customers with $i$ as its index and $n=|I|$.

$J$ : set of potential facilities with $j$ as its index and $m=|J|$.

$p^{\max }:$ the maximum number of open facilities.

$w_{i}$ : the demand of customer $i(i \in I)$

$a_{i}=\left(a_{i}^{1}, a_{i}^{2}\right)$ : location of customer $i$ where $a_{i} \in \mathbb{R}^{2}, i \in I$

$\hat{a}_{j}=\left(\hat{a}_{j}^{1}, \hat{a}_{j}^{2}\right)$ : location of facility $j$ where $\hat{a}_{j} \in \mathbb{R}^{2}, j \in J$

$K$ : the number of potential capacity levels

$F$ : the basic fixed cost for opening a facility

$\lambda_{k}$ : the correction factor for opening a facility with capacity $k(k=1, \ldots, K)$

$F_{k}=\lambda_{k} \cdot F$ : the fixed cost of opening a facility with capacity $k(k=1, \ldots, K)$

$b_{k}$ : the $k^{\text {th }}$ capacity $(k=1, \ldots, K)$

We define the discrete metrics as follow:

$$
\begin{aligned}
& d_{i j}=\left(\left|\hat{a}_{j}^{1}-a_{i}^{1}\right|+\left|\hat{a}_{j}^{2}-a_{i}^{2}\right|\right) \text { for Rectilinear distance } \\
& d_{i j}=\left(\left(\hat{a}_{j}^{1}-a_{i}^{1}\right)^{2}+\left(\hat{a}_{j}^{2}-a_{i}^{2}\right)^{2}\right)^{1 / 2} \text { for Euclidean distance }
\end{aligned}
$$

\section{Decision Variables}

$$
\begin{aligned}
& X_{i j}= \begin{cases}1 & \text { if customer } i(i \in I) \text { is served by facility } j(j \in J), \\
0 & \text { otherwise }\end{cases} \\
& Y_{j k}= \begin{cases}1 & \text { if facility } j \text { uses design } k, \\
0 & \text { otherwise }\end{cases}
\end{aligned}
$$

The problem can be modelled as an integer linear problem as follows. 


$$
\text { Minimise } \sum_{i=1}^{n} w_{i} \sum_{j=1}^{m} X_{i j} \cdot d_{i j}+\sum_{k=1}^{K} F_{k} \sum_{j=1}^{m} Y_{j k}
$$

subject to

$$
\begin{gathered}
\sum_{j=1}^{m} X_{i j}=1, \quad \forall i \in I \\
\sum_{j=1}^{m} \sum_{k=1}^{K} Y_{j k} \leq p^{\max } \\
\sum_{i=1}^{n} w_{i} \cdot X_{i j} \leq \sum_{k=1}^{K} b_{k} \cdot Y_{j k}, \quad \forall j \in J \\
\sum_{k=1}^{K} Y_{j k} \leq 1, \quad \forall j \in J \\
X_{i j} \in\{0,1\}, \quad \forall i \in I, j \in J \\
Y_{j k} \in\{0,1\}, \quad \forall j \in J, k=1, \ldots, K
\end{gathered}
$$

The objective function (1) is to minimise the sum of the total cost consisting of the opening facilities and the transportation costs. Constraints (2) ensure that each demand point is served by one facility. Constraints (3) make sure that the number of open facilities does not exceed the maximum number of open facilities. Constraints (4) guarantee that capacity constraints of the facilities are satisfied. Constraints (5) indicate that each opening facility only use one level of capacity. Constraints (6) and (7) refer to the binary nature of the decision variables.

\section{B. The DLP-CLC}

In the DLP-CLC, both capacity and location costs are taken into account. As the location of potential facilities is fixed, the area of the potential facility is known resulting in the location cost for each potential facility site being known. In the literature, this refers to as site-dependent fixed cost. The formulation of the mathematical model of this problem is similar to the DPL-CC model except the objective function and the following parameters are added:

$\hat{\theta}_{j}$ : the correction factor of opening facility in site $j(j=1, \ldots, m)$

$\hat{F}_{k j}=\hat{\theta}_{j} \cdot \lambda_{k} \cdot F$ : fixed cost of facility with capacity $k$ in site $j(k=1, \ldots, K ; j=$ $1, \ldots, m)$

The problem can be modelled as an integer linear problem as follows

$$
\text { Minimise } \sum_{i=1}^{n} w_{i} \sum_{j=1}^{m} X_{i j} \cdot d_{i j}+\sum_{j=1}^{m} \sum_{k=1}^{K} \hat{F}_{j k} \cdot Y_{j k}
$$

with decision variables and constraints being the same as in the previous model (i.e., Equations 17). 


\subsection{Continuous Location Problems}

Similar to the discrete problem, we also propose two models namely the Continuous Location Problem with Capacity Cost (CLC-CC), and the Continuous Location Problem with Capacity and Location Costs (CLP-CLC).

\section{A. The CLP-CC}

The CLP-CC is the continuous version of its counterpart the DLP-CC. Sets and parameters are similar to the ones used in the discrete model except that the set of potential facilities is not needed here and the following new decision variables are used:

\section{Decision Variables}

$$
\begin{aligned}
X_{i j} & = \begin{cases}1 & \text { if customer } i(i \in I) \text { is served by facility } j\left(j=1, \ldots, p^{\max }\right), \\
0 & \text { otherwise }\end{cases} \\
Y_{j k} & = \begin{cases}1 & \text { if facility } j \text { uses design } k \\
0 & \text { otherwise }\end{cases} \\
C_{j} & =\left(c_{j}^{1}, c_{j}^{2}\right): \text { coordinate of facility } j \text { where } C_{j} \in \mathbb{R}^{2}
\end{aligned}
$$

We define the following distance metrics as follow:

$$
\begin{aligned}
& d_{i j}=\left(\left|c_{j}^{1}-a_{i}^{1}\right|+\left|c_{j}^{2}-a_{i}^{2}\right|\right) \text { for Rectilinear distance } \\
& d_{i j}=\left(\left(c_{j}^{1}-a_{i}^{1}\right)^{2}+\left(c_{j}^{2}-a_{i}^{2}\right)^{2}\right)^{1 / 2} \text { for Euclidean distance }
\end{aligned}
$$

The problem can be modelled as a nonlinear problem as follows.

$$
\text { Minimise } \sum_{i=1}^{n} w_{i} \sum_{j=1}^{p^{\max }} X_{i j} \cdot d_{i j}+\sum_{k=1}^{K} F_{k} \sum_{j=1}^{p^{\max }} Y_{j k}
$$

subject to

$$
\begin{aligned}
& \sum_{j=1}^{p^{\max }} X_{i j}=1, \quad \forall i \in I \\
& \sum_{i=1}^{n} w_{i} \cdot X_{i j} \leq \sum_{k=1}^{K} b_{k} \cdot Y_{j k}, \quad \forall j=1, \ldots, p^{\max } \\
& \sum_{k=1}^{K} Y_{j k} \leq 1, \quad \forall j=1, \ldots, p^{\max } \\
& X_{i j} \in\{0,1\}, \quad \forall i \in I, j=1, \ldots, p^{\max } \\
& Y_{j k} \in\{0,1\}, \quad \forall j=1, \ldots, p^{\max }, k=1, \ldots, K \\
& C_{j} \in \mathbb{R}^{2}, \quad \forall j=1, \ldots, p^{\max }
\end{aligned}
$$

The objective function (9) aims to minimise the sum of the total costs including the opening facilities and the transportation costs. Constraints (10) guarantee 
that each demand point is served by one facility. Constraints (11) ensure that capacity constraints of the facilities are met. Constraints (12) state that each opening facility only uses one capacity design. Constraints (13) and (14) refer to the binary nature of the decision variables while Constraints (15) indicate the continuous location variables.

\section{B. The CLP-CLC}

This model is an enhancement of the model proposed by Irawan et al (2017). In the DLP-CLC, the opening facility cost is not only based on the chosen capacity but also on the region/zone where the facility is sited. In this model, a number of areas are given along with their cost. As the location of facilities is unknown, the region/zone is also treated as a decision variable. For simplicity, we consider the shape of each zone to be a convex polygon. We use the D-function to check whether a point is inside a convex polygon or not, see Chernov et al (2009) and Irawan et al (2017). For simplicity this mechanism is as follows. The direction of the edges that makes up the zone is the same (clockwise or anticlockwise) where Figure 1a presents an example of an area. Each edge vector of the polygon has four parameters $(\alpha, \beta, \gamma$, and $\delta)$ which are defined in Figure $1 \mathrm{~b}$.

A point, say point $P_{3}\left(x_{3}, y_{3}\right)$, is inside the polygon if this point is on the right hand side of all edges. The check is performed to see whether a point is on the right side of an edge. Let $\theta=\alpha \cdot x_{3}+\beta \cdot y_{3}+\gamma$. Point $P_{3}$ lies on the edge if $\theta=0$. In case that $\theta>0$, point $P_{3}$ is on the left hand side of the edge, otherwise point $P_{3}$ will be on the right hand side of the edge.

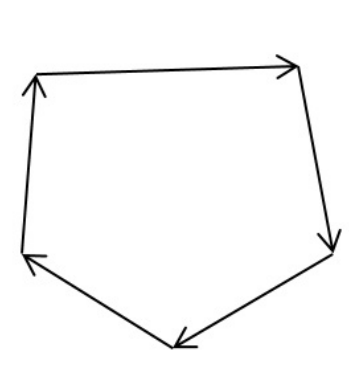

(a)

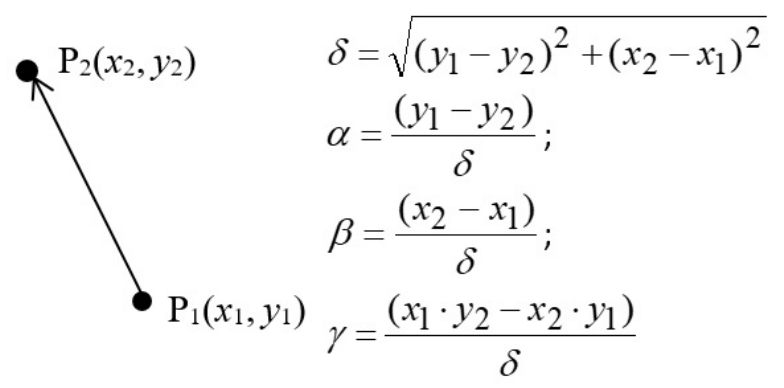

(b)

Fig. 1 An example of a convex polygon with parameters formulation of the edges

\section{Sets and Parameters}

These are similar to the previous model except the following:

$R$ : set of areas with $r$ as its index

$E_{r}$ : set of edges of area $r(r \in R)$ with $e$ as its index. The edges made up a convex polygon (area) where one or more facilities can be located

$O$ : the number of areas (in this study, the area shape is convex polygon)

$\alpha_{e r}$ : the parameter used to formulate Edge $e\left(e \in E_{r}\right)$ 
$\beta_{e r}$ : the parameter used to formulate Edge $e\left(e \in E_{r}\right)$

$\gamma_{e r}$ : the parameter used to formulate Edge $e\left(e \in E_{r}\right)$

$\theta_{r}$ : the correction factor of opening facility in area $r(r=1, \ldots, O)$

$\tilde{F}_{k r}=\theta_{r} \cdot \alpha_{k} \cdot F$ : fixed cost of facility with capacity $k$ in area $r(k=1, \ldots, K ; r=$ $1, \ldots, O)$

Decision Variables

$X_{i j}= \begin{cases}1 & \text { if customer } i(i \in I) \text { is served by facility } j\left(j=1, \ldots, p^{\max }\right), \\ 0 & \text { otherwise }\end{cases}$

$Y_{j k r}= \begin{cases}1 & \text { if facility } j \text { located in area } r \text { uses capacity } k, \\ 0 & \text { otherwise }\end{cases}$

$C_{j}=\left(c_{j}^{1}, c_{j}^{2}\right)$ : coordinate of facility $j$ where $C_{j} \in \mathbb{R}^{2}$

The problem can be modelled as a nonlinear problem as follows.

$$
\text { Minimise } \sum_{j=1}^{p^{\max }} \sum_{i=1}^{n} w_{i} \cdot X_{i j} \cdot d_{i j}+\sum_{j=1}^{p^{\max }} \sum_{k=1}^{K} \sum_{r=1}^{O} \tilde{F}_{k r} \cdot Y_{j k r}
$$

subject to

$$
\begin{gathered}
\sum_{j=1}^{p_{\max }} X_{i j}=1, \quad \forall i \in I \\
\sum_{i=1}^{n} w_{i} \cdot X_{i j} \leq \sum_{k=1}^{K} \sum_{r=1}^{O} b_{k} \cdot Y_{j k r}, \quad \forall j=1, \ldots, p^{\max } \\
\sum_{k=1}^{K} \sum_{r=1}^{O} Y_{j k r} \leq 1, \quad \forall j=1, \ldots, p^{\max } \\
\forall r \in R, e \in E_{r}, k=1, \ldots, K, j=1, \ldots, p^{\max } \\
\alpha_{e r} \cdot c_{j}^{1}+\beta_{e r} \cdot c_{j}^{2}+\gamma_{e r} \leq U \cdot\left(1-Y_{j k r}\right), \\
Y_{j k r} \in\{0,1\}, \quad \forall i \in I, j=1, \ldots, p^{\max } \\
C_{j} \in \mathbb{R}^{2}, \quad \forall j=1, \ldots, p^{\max }, k=1, \ldots, K
\end{gathered}
$$

where

$$
U=\max _{i}\left(\operatorname{Max}\left(a_{i}^{1}, a_{i}^{2}\right)\right)
$$

The objective function (16) is to minimise the sum of the total costs including the location cost. Constraints (20) indicate the area of the open facilities.

\section{Linearisation of the continuous model}

In case the rectilinear distance is used, the above models can be linearized as follows: 
First we define $a_{\min }^{1}=\min _{i}\left(a_{i}^{1}\right), a_{\max }^{1}=\max _{i}\left(a_{i}^{1}\right), a_{\min }^{2}=\min _{i}\left(a_{i}^{2}\right), a_{\max }^{2}=$ $\max _{i}\left(a_{i}^{2}\right)$, and set $\hat{a}_{\min }^{1}=0, \hat{a}_{\max }^{1}=a_{\max }^{1}-a_{\min }^{1}, \hat{a}_{\min }^{2}=0$, and $\hat{a}_{\max }^{2}=a_{\max }^{2}-$ $a_{\min }^{2}$. We then transform the coordinate of each customer by $\hat{a}_{i}=\left(a_{i}^{1}-a_{\min }^{1}, a_{i}^{2}-a_{\min }^{2}\right)$ which will be used in the mathematical model.

Given that

$$
X_{i j} \cdot w_{i} \cdot\left(\left|c_{j}^{1}-\hat{a}_{i}^{1}\right|+\left|c_{j}^{2}-\hat{a}_{i}^{2}\right|\right)=w_{i} \cdot\left(\left|X_{i j} \cdot c_{j}^{1}-X_{i j} \cdot \hat{a}_{i}^{1}\right|+\left|X_{i j} \cdot c_{j}^{2}-X_{i j} \cdot \hat{a}_{i}^{2}\right|\right)
$$

We define the new variables as $\rho_{i j}^{1}=X_{i j} \cdot c_{j}^{1}$ and $\rho_{i j}^{2}=X_{i j} \cdot c_{j}^{2}$.

The first part of the objective function in Equations (9) and (16) can then be rewritten as follow:

$$
\text { Minimise } \sum_{i=1}^{n} w_{i} \cdot \sum_{j=1}^{p^{\max }}\left(\left|\rho_{i j}^{1}-X_{i j} \cdot \hat{a}_{i}^{1}\right|+\left|\rho_{i j}^{2}-X_{i j} \cdot \hat{a}_{i}^{2}\right|\right)
$$

We now have a linear model with the following additional constraints:

$$
\begin{gathered}
\rho_{i j}^{1} \leq \hat{a}_{\max }^{1} \cdot X_{i j}, \quad \forall i \in I, j=1, \ldots, p^{\max } \\
\quad \rho_{i j}^{1} \geq \hat{a}_{\min }^{1} \cdot X_{i j}, \quad \forall i \in I, j=1, \ldots, p^{\max } \\
\rho_{i j}^{1} \leq c_{j}^{1}-\hat{a}_{\min }^{1} \cdot\left(1-X_{i j}\right), \quad \forall i \in I, j=1, \ldots, p^{\max } \\
\rho_{i j}^{1} \geq c_{j}^{1}-\hat{a}_{\max }^{1} \cdot\left(1-X_{i j}\right), \quad \forall i \in I, j=1, \ldots, p^{\max } \\
\rho_{i j}^{2} \leq \hat{a}_{\max }^{2} \cdot X_{i j}, \quad \forall i \in I, j=1, \ldots, p^{\max } \\
\rho_{i j}^{2} \geq \hat{a}_{\min }^{2} \cdot X_{i j}, \quad \forall i \in I, j=1, \ldots, p^{\max } \\
\rho_{i j}^{2} \leq c_{j}^{1}-\hat{a}_{\min }^{2} \cdot\left(1-X_{i j}\right), \quad \forall i \in I, j=1, \ldots, p^{\max } \\
\rho_{i j}^{2} \geq c_{j}^{1}-\hat{a}_{\max }^{2} \cdot\left(1-X_{i j}\right), \quad \forall i \in I, j=1, \ldots, p^{\max }
\end{gathered}
$$

As $\hat{a}_{\min }^{1}=\hat{a}_{\min }^{2}=0$, we can substitute this value in constraints (26), (27), (30), and (31) making the above model more concise.

\section{Solution methods}

Based on our preliminary study, the location models with opening cost for discrete and continuous cases are very hard to solve using an exact method such as CPLEX especially when the size of the problem is large $(n \geq 500)$. To overcome this weakness, two solution methods that incorporates the exact method and heuristic technique are proposed. Such a hybridisation is usually powerful and known as matheuristics (see Salhi (2017) for more details). The exact method is used to optimally solve the customer allocation problem whenever the locations of the opening facilities are fixed. Note that when the number of open facilities $(\hat{p})$ and the location of open facilities $C_{j}\left(c_{j}^{1}, c_{j}^{2}\right)$ are known, the problem reduces to an assignment problem. The model will also find the optimal capacity for each opening facility. Here the distance between a facility and a customer $\left(d_{i j}\right)$ is treated as an input instead of a decision variable. The formulation of the integer linear model is presented as follows. 


\section{Decision Variables}

$$
\begin{aligned}
& X_{i j}= \begin{cases}1 & \text { if customer } i(i \in I) \text { is served by facility } j(j \in J), \\
0 & \text { otherwise }\end{cases} \\
& Y_{j k}= \begin{cases}1 & \text { if facility } j \text { uses capacity } k, \\
0 & \text { otherwise }\end{cases}
\end{aligned}
$$

The objective function of the problem with capacity cost:

$$
\text { Minimise } \sum_{i=1}^{n} w_{i} \cdot \sum_{j=1}^{\hat{p}} X_{i j} \cdot d_{i j}+\sum_{k=1}^{K} F_{k} \cdot \sum_{j=1}^{\hat{p}} Y_{j k}
$$

The objective function of the problem with capacity and location costs:

$$
\text { Minimise } \sum_{i=1}^{n} w_{i} \cdot \sum_{j=1}^{\hat{p}} X_{i j} \cdot d_{i j}+\sum_{j=1}^{\hat{p}} \sum_{k=1}^{K} \hat{F}_{k j} \cdot Y_{j k}
$$

subject to

$$
\begin{gathered}
\sum_{j=1}^{\hat{p}} X_{i j}=1, \quad \forall i \in I \\
\sum_{j=1}^{\hat{p}} \sum_{k=1}^{K} Y_{j k}=\hat{p} \\
\sum_{i=1}^{n} w_{i} \cdot X_{i j} \leq \sum_{k=1}^{K} b_{k} \cdot Y_{j k}, \quad \forall j \in J \\
\sum_{k=1}^{K} Y_{j k}=1, \quad \forall j \in J \\
X_{i j} \in\{0,1\}, \quad \forall i \in I, j \in J \\
Y_{j k} \in\{0,1\}, \quad \forall j \in J, k=1, \ldots, K
\end{gathered}
$$

Constraints (36) ensure that all the facilities are open whereas (38) guarantee that a customer is served by one facility with a given capacity.

In this section, the two proposed solution methods are described. The proposed approaches are used to solve both discrete and continuous problems. The first method is referred to as an iterative matheuristic approach whereas the second one is called VNS-based matheuristic. In the second approach, a powerful metaheuristic technique known as Variable Neighbourhood Search (VNS) is implemented. 
4.1 The iterative matheuristic approach (IMA)

The main steps of the proposed iterative matheuristic approach (IMA) are presented in Algorithm 1. The method is an iterative approach where the algorithm seeks the best solution for various values of the number of the open facilities starting from $p^{\min }$ to $p^{\max }$ where $p^{\text {min }}$ is calculated based on the total demand and the maximum capacity used by an open facility. The algorithm starts from $p=p^{\text {min }}$ where the initial solution (the location of opening facilities) is randomly generated. When $p>p^{\mathrm{min}}$, the initial solution is based on the best solution obtained when the number of opening facilities is set to $(p-1)$ with an additional randomly generated opening facility location. In other words, the set of facility locations obtained from the previous iteration is treated as the promising potential sites. Moreover, this procedure is known as a learning process where the result from a current iteration will be fed into the next one. In Step 3, a multi-start procedure is applied where an iterative process is conducted until the number of iterations reaches a prescribed maximum number of iterations $(T)$. This can be easily performed as our model (33) - (40) is relatively easy to solve using the exact method by CPLEX though any other powerful software can be used.

Algorithm 1: The iterative matheuristic for both discrete and continuous problems

Initialization:

(a) Let $x_{j}^{*}$ and $y_{j}^{*}$ be $\mathrm{x}$ - and $\mathrm{y}$ - coordinates of the location of open facility $j\left(j=1, \ldots, p^{*}\right)$ in the best solution with $\mathbb{k}_{j}^{*}$ be the capacity of the facility and $z^{*}$ be its objective function value

(b) Calculate $p^{\min }=\left\lfloor\frac{\sum_{i \in I} w_{i}}{\max _{k}\left(b_{k}\right)}\right\rfloor$ and set $p=p^{\min }$

(c) Define the number of iterations $(T)$ and set $z^{*}=\infty$

\section{Main Steps:}

Repeat the following steps until $p>p^{\max }$

Step 1: Let $\left(\hat{x}_{p j}, \hat{y}_{p j}\right)$ be the coordinates of the location of the open facility $j$ in the best solution when the number of open facilities is set to $p$. Denote $\hat{\mathbb{k}}_{p j}$ be the capacity of facility $j(j=1, \ldots, p)$ and $\hat{z}_{p}$ be its objective function value

Step 2: Set $\hat{z}=\infty$

Step 3: For iter $=1$ to $T$ do the following steps:

(a) If $p=p^{\text {min }}$ then pick randomly $p$ customer sites as the location of the open facilities with $\left(x_{p j}, y_{p j}\right)$ as their coordinates $j(j=1, \ldots, p)$

Else take the best facility location obtained when the number of open facilities is set to $(p-1)$. In other words, $x_{p j}=\hat{x}_{(p-1) j}$ and $y_{p j}=\hat{y}_{(p-1) j}$, $j=1, \ldots,(p-1)$. To complement the number of open facilities, choose randomly a customer location as an open facility location

(b) Solve the allocation problem given by model (33)-(40) using CPLEX with the location of open facilities $\left(x_{p j}, y_{p j}\right)$ as an input. Let $z_{p}$ be its objective function value and $\mathbb{k}_{p j}$ be the capacity of facility $j(j=1, \ldots, p)$

(c) This step is for continuous problem: Call a local search procedure given in Algorithm 3 with $\left(x_{p j}, y_{p j}\right)$ as an input to improve the solution. As result, the value of $z_{p}, x_{p j}$ and $y_{p j}$ may change.

(d) If $z_{p}<\hat{z}_{p}$ then $\hat{z}_{p} \leftarrow z_{p}, \hat{x}_{p j} \leftarrow x_{p j}, \hat{y}_{p j} \leftarrow y_{p j}$, and $\hat{\mathbb{k}}_{p j} \leftarrow \mathbb{k}_{p j}$. 
Step 4: This step is for discrete problem: Improve the quality of solution $\left(\hat{z}_{p}\right)$ using the proposed local search presented in Algorithm 2 with $\left(\hat{x}_{p j}, \hat{y}_{p j}\right)$ as an input.

Step 5: If $\hat{z}_{p}<z^{*}$ then $z^{*} \leftarrow \hat{z}_{p}, p^{*} \leftarrow p, x_{j}^{*} \leftarrow \hat{x}_{p j}, y_{j}^{*} \leftarrow \hat{y}_{p j}$, and $\mathbb{k}_{j}^{*} \leftarrow \hat{\mathbb{k}}_{p j}$.

In this study, a similar but tailored local search for both the discrete and continuous cases is used. The algorithm will seek the smallest objective function value $\left(z^{*}\right)$ along with the corresponding number of opening facilities $\left(p^{*}\right)$ with their respective coordinates $\left(x_{j}^{*}, y_{j}^{*}\right)$ for $j=1, \ldots, p^{*}$.

\section{Local search for the discrete problem}

The proposed local search procedure for the discrete problem is presented in Algorithm 2 where the initial objective function value $(z)$, the number of open facilities $(p)$ with then initial locations $\left(x_{j}, y_{j}\right)$ for $j=1, \ldots, p$ are given as inputs. This local search is based on the interchange heuristic using the first improvement strategy. In Step 2(b), the location of facility $j$ is moved to the location of customer $i$ where customer $i$ is served by facility $j$. In other words, the new location of facility $j$ will be relatively close to the old one for reducing the computing time. Model (33)-(40) is solved to calculate the objective function value and for allocating customers to the open facilities. As the first improvement is applied, once the swapping process improves the solution, the location of a facility moves to the customer site. This procedure is repeated until there no improvement is found or the maximum time allowed for the local search $\left(t_{\max }\right)$ is reached.

Algorithm 2: The proposed local search for the discrete problem

Input: $z, p, x_{j}$, and $y_{j}$

Initialization: Define $t_{\max }$ and compute $N_{j}(j=1, \ldots, p)$

Step 1: Set $\tau=$ true

Step 2:

(a) Set $\theta_{\text {best }}=0$ ( $\theta_{\text {best }}$ is the best saving occurred from the swapping operator).

(b) For $j=1$ to $p$ do the following steps:

For each customer $i \in N_{j}$ do the following:

- Move the location of facility $j$ to the location of customer $i$

- Solve optimally the allocation problem by solving model (33)-(40) with the new location of facility $j$. Let $\tilde{z}$ be its objective function value.

- Calculate the saving $\theta=z-\tilde{z}$

- If $\theta>\theta_{\text {best }}$, set $\theta_{\text {best }} \leftarrow \theta, j_{\text {best }} \leftarrow j, i_{\text {best }} \leftarrow i$, and go to Step 3

- If CPU time is greater than $t_{\max }$, update $\tau=$ false and go to Step 3

Step 3:

(a) If $\theta_{\text {best }}>0$, update $z \leftarrow z-\theta_{\text {best }}, x_{j_{\text {best }}} \leftarrow a_{i_{\text {best }}}^{1}$, and $y_{j_{\text {best }}} \leftarrow a_{i_{\text {best }}}^{2}$.

(b) If $\theta_{\text {best }} \leq 0$ or $\tau=$ false, stop, otherwise compute $N_{j}(j=1, \ldots, p)$ and go to Step 2. 


\section{Local search for the continuous problem}

Algorithm 3 presents the main steps of our proposed local search for the continuous problem. The input for this procedure is identical to the one for the discrete problem. In the proposed local search, ALA is implemented where the Weiszfelds formula (see Equation 41) is used to find the new location of the open facilities. In other words, the current location of an open facility, $C_{j}\left(x_{j}, y_{j}\right)$, is updated into a new location $\tilde{C}_{j}\left(\tilde{x}_{j}, \tilde{y}_{j}\right)$ using $(41)$,

$$
\tilde{x}_{j}=\frac{\sum_{i \in N_{j}} \frac{w_{i} \cdot a_{i}^{1}}{d\left(C_{j}, a_{i}\right)}}{\sum_{i \in N_{j}} \frac{w_{i}}{d\left(C_{j}, a_{i}\right)}} ; \tilde{y}_{j}=\frac{\sum_{i \in N_{j}} \frac{w_{i} \cdot a_{i}^{2}}{d\left(C_{j}, a_{i}\right)}}{\sum_{i \in N_{j}} \frac{w_{i}}{d\left(C_{j}, a_{i}\right)}}
$$

where $N_{j}$ is the set of customers that is served by facility $j$ and $d\left(C_{j}, a_{i}\right)$ is the distance between facility $j$ and customer $i$.

The model (33)-(40) is then solved considering the new facility locations resulting in a new objective function value $(\tilde{z})$ and customer allocations. This process is repeated until the gap of the objective function value between the current iteration and the previous one in the ALA algorithm is less than $\varepsilon$.

Algorithm 3: The proposed local search for the continuous problem

Input: $z, p, x_{j}$, and $y_{j}$

Initialization: Define $\varepsilon$ and compute $N_{j}(j=1, \ldots, p)$

\section{Main Step}

Step 1:

Update $\tilde{x}_{j} \leftarrow x_{j}$ and $\tilde{y}_{j} \leftarrow y_{j}$ using Weiszfelds (41)

Step 2:

Solve the allocation problem by solving model (33)-(40) with the location of open facilities with $\left(\tilde{x}_{j}, \tilde{y}_{j}\right)$ as an input. Let $\tilde{z}$ be its objective function value and $N_{j}$ the set of customers that are served by facility $j, j=1, \ldots, p$

Step 3:

If $(z-\tilde{z}) \leq \varepsilon$, stop and return the values of $z, x_{j}$, and $y_{j}$

Else update $z \leftarrow \tilde{z}, x_{j} \leftarrow \tilde{x}_{j}, y_{j} \leftarrow \tilde{y}_{j}$ and go back to Step 1 .

\subsection{The VNS-based matheuristic approach}

Variable neighbourhood search (VNS) was introduced by Brimberg and Mladenović (1996) for solving continuous location-allocation problems. However, Hansen and Mladenović (1997) first formally formulated this metaheuristic when solving the $p$-median problem. For more details on VNS implementations and its variants, see Hansen and Mladenović (2001) and Hansen et al (2010). VNS consists of local search and neighbourhood search where the former seeks local optimality whereas the latter aims to escape from these local optima by systematically using a larger neighbourhood if no improvement is found otherwise, the search reverts back to the first neighborhood, usually the smallest one. The main steps 
of our VNS based matheuristic are presented in Algorithm 4. In the first step, the parameters required in the proposed matheuristic method are also defined here including the maximum computational time $\left(c p u_{\max }\right)$ and the number of neighborhood structures $\left(k_{\max }\right)$.

Algorithm 4: The procedure of the proposed VNS-based matheuristic

Initialization:

(a) As the initialisation step of Algorithm 1

(b) Define $\sigma, \eta, c p u_{\max }$ and $k_{\max }$

Stage 1: finding a good initial solution

1. Set $p=p_{\min }$ and $p^{\text {hi }}=p^{\max }$

2. Repeat the following steps until $p>p^{\max }$

(a) Apply steps 1-3 of Algorithm 1. Note that Step 1c) is also carried out for discrete problem

(b) Store the values of $\hat{z}_{p}, \hat{x}_{p j}, \hat{y}_{p j}$, and $\hat{\mathbb{k}}_{p j}$

(c) If there is no improvement within $\sigma$ successive times (in term of $\hat{z}_{p}$ ) then set $p^{\text {hi }}=p$ and go to Step 3

(d) $p=p+1$

3. Set $p=p^{\text {hi }}-1$

4. Repeat the following steps until $p<p^{\text {min }}$

(a) Apply steps 2a)-2b). Note that in Step 1a), we take the best facility location obtained when the number of open facilities is set to $(p+1)$. Then, we remove randomly one facility from this configuration

(b) Store the values of $\hat{z}_{p}, \hat{x}_{p j}, \hat{y}_{p j}$, and $\hat{\mathbb{k}}_{p j}$

(c) If there is no improvement within $\sigma$ successive times then go to Step 5

(d) $p=p-1$

5. Take $\eta$ best facility configurations with different values of $p$ from previous steps

6. For continuous problem: Use the local search in Algorithm 3 starting from $\eta$ different initial solutions based on different values of $p$.

For discrete problem: For each facility from the best facility configurations do:

(a) Move the facility site (continuous) to the nearest potential facility site (discrete)

(b) Use the local search in Algorithm 2 using the facility configuration obtained from the previous step (a) as the initial solution

7. Take the best number of facilities $\left(p^{*}\right)$, facility configuration $S *\left(x_{j}^{*}, y_{j}^{*}\right)$, capacity configuration $\left(\mathbb{k}_{j}^{*}\right)$ and objective function value $\left(z^{*}\right)$

Stage 2: VNS Implementation

8. Set $k=1$

9. Set $S \leftarrow S^{*}$

10. Shaking Procedure

Do the following step $k$ times:

(a) Choose randomly a customer site, say customer $\hat{i}$

(b) Change a facility (that serve customer $\hat{i}$ ), say facility $\hat{j}$, from the current solution $\left(S^{\prime}\right)$ with the location of customer $\hat{i}$ without changing its capacity 
(c) Implement CPLEX to solve the assignment problem (GAP) based on $\left(S^{\prime}\right)$ and capacity configuration $\left(\mathbb{k}_{j}^{*}\right)$ with $z^{\prime}$ as its objective function value

11. Local search

For discrete problem: Implement the proposed local search presented in Algorithm 2 with $z^{\prime}$ and $S^{\prime}\left(x_{j}^{\prime}, y_{j}^{\prime}\right)$ as inputs and outputs.

For continuous problem: Apply the proposed local search given in Algorithm 3 with $z^{\prime}$ and $S^{\prime}\left(x_{j}^{\prime}, y_{j}^{\prime}\right)$ as inputs and outputs

12. Move or Not

If $z^{\prime}<z^{*}$ then update $k=1$ along with $z^{*}=z^{\prime}$ and $S^{*} \leftarrow S^{\prime}$

Else update $k=k+1$ along with $z^{\prime}=z^{*}$ and $S^{\prime} \leftarrow S^{*}$

13. If computing time is greater than $c p u_{\max }$ then go to Step 15

14. If $k \leq k_{\max }$ go back to Step 10

15. Return $z^{*}, S^{*}, p^{*}, \mathbb{k}_{j}^{*}$

In the first stage, an iterative procedure to find a good initial solution is put forward. This stage aims to seek the best number of open facilities $\left(p^{*}\right)$ along with its facility configuration (location and capacity). Step 2 of Algorithm 4 is similar to Step 1-3 of Algorithm 1. It finds the best solution for various $p$ starting from $p_{\min }$ to $p_{\max }$ in the continuous space for both discrete and continuous problems. The search terminates when there is no improvement within $\sigma$ successive times. To speed up the search, in Step 3c), we fixed the capacity of each facility when solving allocation problem (Equations 33-40). In Step 4 of Algorithm 4, similarly to Step 2, seeking the best configuration is also conducted but starting from higher $p\left(p^{\text {hi }}\right)$ to $p_{\text {min }}$. Here, the initial solution is based on the best solution obtained when the number of opening facilities is set to $(p+1)$. Then a facility is randomly removed. The proposed local searches (for both discrete and continuous problems) are applied starting from the best facility configurations with different values of $\mathrm{p}$ found in the previous steps. In case of discrete problem, before the proposed local search is implemented, in the initial solution we move the facility sites to the nearest potential facility sites (customer sites). To speed up the search, in the local searches we fix the capacity of each facility when solving the allocation problem. From the results of local searches, we take the best number of facilities $\left(p^{*}\right)$, facility configuration $S^{*}\left(x_{j}^{*}, y_{j}^{*}\right)$, capacity configuration $\left(\mathbb{k}_{j}^{*}\right)$ and objective function value $\left(z^{*}\right)$. This solution is then fed into the next stage, which is the VNS algorithm.

In the proposed VNS, the shaking process (Step 10) is carried out by choosing randomly a customer site, say customer $\hat{i}$, and moving the location of the facility that serves customer $\hat{i}$ to this customer site. Note that, the capacity for the facility does not change. The quality of the new facility is evaluated by solving the allocation problem (Equations 33-40). This process is repeated $k$ times. In Step 11, the proposed local searches (Algorithms 2 and 3 ) are put forward to improve the quality of solution by finding the local optima. In the local search, we fix the capacity of each facility when solving the allocation problem. This restriction aims to reduce the computational time at the expense of a small loss in terms of solution quality. Step 12 of the algorithm is the Move or Not step where if the local search is not able to improve the solution, a larger neighborhood is gradually utilised otherwise the smallest one will be used. This can be carried out by updating the 
value of $k$ with $k=k_{\text {max }}$ representing the index of the largest neighborhood and $k=1$ the index of the smallest one. In VNS, it is claimed that the smallest neighborhood is the one that is closest to the current solution, whereas the largest one is the farthest from the current solution (Hansen and Mladenović, 1997).

\section{Computational Experiments}

Extensive experiments to examine the performance of the proposed matheuristic approaches were carried out. The algorithms were coded in C++ .Net 2012 and the IBM ILOG CPLEX version 12.6 Concert Library is used for solving the models with the exact method. The experiments were run on a PC with an Intel Core i5 CPU @ 3.20GHz processor, 8.00 GB of RAM and under Windows 7. The performance of the proposed methods was assessed using generated dataset constructed based on the four well known data sets from Brimberg et al (2000) originally used for the multi-facility Weber problem with the addition of setting various capacities and location costs. It consists of 50, 287, 654, and 1060 customers with the demand of all data sets being unity except the one with 287 customers.

The possible capacity levels along with the capacity and the corresponding opening cost is presented in Table 1. For the opening cost related to the site where the facilities are located, each region/zone is divided into two types of polygons namely the hexagonal and the diamond shapes where they are referred to as the diamond and the hexagonal data sets. For illustration, Figure 2 shows an example of the diamond and the hexagonal datasets with $n=50$. The area is categorized into three classifications namely light grey, medium grey, and dark grey areas with three setting of $\theta_{r}(1,1.25$, and 1.5$)$ respectively.

Table 1 The possible design capacities with its opening cost

\begin{tabular}{|c|c|c|c|}
\hline$n$ & Capacity Design $(k)$ & Capacity & $\operatorname{Cost}\left(F_{k}\right)$ \\
\hline \multirow{3}{*}{50} & 1 & 2 & 4 \\
\hline & 2 & 6 & 6 \\
\hline & 3 & 10 & 7.5 \\
\hline \multirow{3}{*}{287} & 1 & 500 & 500 \\
\hline & 2 & 750 & 700 \\
\hline & 3 & 1000 & 850 \\
\hline \multirow{3}{*}{654} & 1 & 50 & 12500 \\
\hline & 2 & 75 & 16000 \\
\hline & 3 & 100 & 20000 \\
\hline \multirow{3}{*}{1060} & 1 & 90 & 105000 \\
\hline & 2 & 120 & 125000 \\
\hline & 3 & 160 & 160000 \\
\hline
\end{tabular}

In our computational experiments, we set the value of $p^{\max }$ to 15 . In the case of the discrete problem, the customer sites are treated as the potential facility sites (i.e., $m=n$ ). To assess our proposed matheuristic techniques, we compare the solutions of the proposed methods with those of the exact method using CPLEX. As the problems are quite hard to solve, we limit the computational time (CPU time) to the one recorded in the exact method. Therefore, lower bound $(L B)$ 

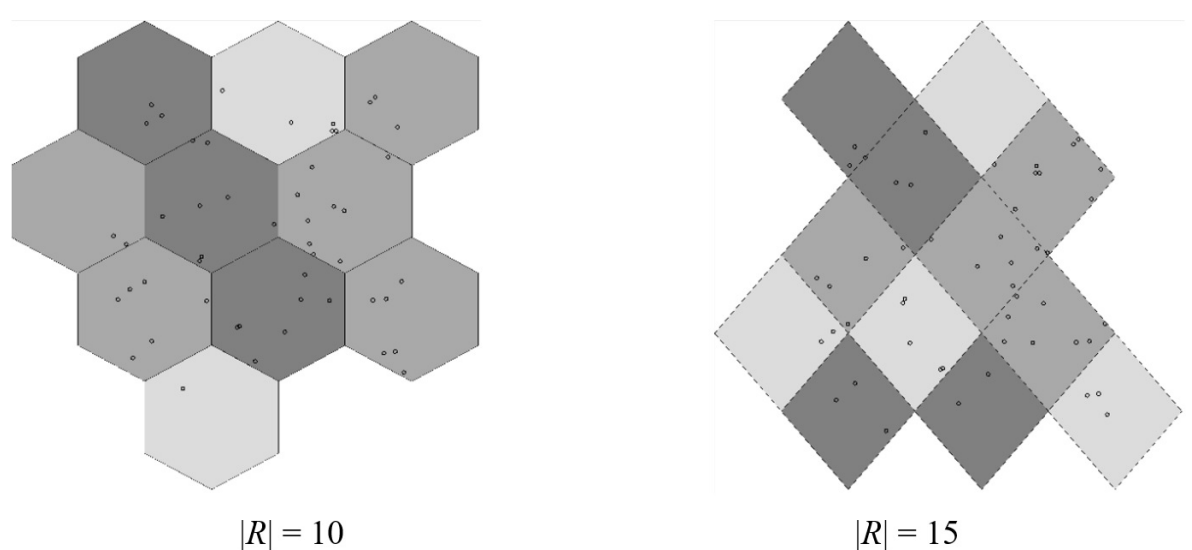

Fig. 2 The shape of area for dataset with $n=50$

and upper bound $(U B)$ are also reported here. The performance of the proposed matheuristic methods are also measured by duality gap denoted by \%gap which is calculated as follows:

$$
\% \text { Gap }=\frac{Z_{h}-L B}{Z_{h}} \times 100
$$

where $Z_{h}$ refers to the objective function value obtained by the proposed matheuristics (or upper bound by the exact method) and lower bound attained from the exact method. The CPU time is measured in seconds.

\subsection{Discrete problem scenario}

The exact methods provided in Section 3 are implemented using CPLEX to first solve the discrete case namely the DLP-CC and the DLP-CLC problems. As the models are relatively hard to solve, we limit the computing time of CPLEX to 3 hours. For the proposed iterative matheuristic approach (IMA), we set the number of iterations $(T)$ to 10 . For the VNS-based matheuristic we set $k_{\max }=p^{*}$. The computational results for the DLP-CC problem are given in Table 2 whereas the results of the DLP-CLC problem using the diamond and the hexagonal data sets are shown in Table 3 and Table 4, respectively. Based on the average gap obtained by the exact method, the complexity of the DLP-CC and DLP-CLC problems is relatively the same. This is due to the opening cost related to the location of potential facilities in the DLP-CLC problem being known.

Based on our computational results given in Table 2, it can be observed that for the smallest instance $(n=50)$, the optimal solution can be obtained using CPLEX for both Rectilinear and Euclidean cases. However, for relatively larger instances ( $n=287$ to 1060), CPLEX was not able to guarantee optimality after the set time of 3 hours. It was also observed that \%Gap increases exponentially with the size of the problem. For $n=50$, IMA found the optimal solution for both Rectilinear and Euclidean cases. On average, IMA produced better solutions for Rectilinear case (\%Gap of 9.28\%) whereas VNS performed better for Euclidean 
case (\%Gap of $9.49 \%$ ) which are relatively much smaller than the ones found by the exact method (15.20\% and $14.58 \%)$. In general, the matheuristic approach runs relatively much faster than the exact method while producing better solutions.

Table 2 Computational results for the DLP-CC

\begin{tabular}{|c|c|c|c|c|c|c|c|c|c|}
\hline \multirow{2}{*}{$n$} & \multicolumn{3}{|c|}{ Exact Method } & \multicolumn{3}{|c|}{ IMA } & \multicolumn{3}{|c|}{ VNS } \\
\hline & $L B$ & Gap (\%) & $p^{*}$ & Gap (\%) & CPU Time & $p^{*}$ & Gap (\%) & CPU Time & $p^{*}$ \\
\hline \multicolumn{10}{|c|}{ Rectilinear Distance } \\
\hline 50 & 112.38 & 0.00 & 10 & 0.00 & 28.21 & 10 & 0.16 & 38.78 & 11 \\
\hline 287 & $13,714.44$ & 3.03 & 14 & 3.15 & $1,473.64$ & 14 & 3.34 & 848.56 & 14 \\
\hline 654 & $250,298.28$ & 15.71 & 13 & 11.19 & $3,305.23$ & 11 & 11.19 & $1,742.13$ & 11 \\
\hline 1060 & $2,074,296.67$ & 42.05 & 15 & 22.79 & $5,418.40$ & 12 & 22.84 & $3,231.47$ & 13 \\
\hline Aver & age & 15.20 & & 9.28 & $2,556.37$ & & 9.38 & $1,465.23$ & \\
\hline \multicolumn{10}{|c|}{ Euclidean Distance } \\
\hline 50 & 101.07 & 0.00 & 10 & 0.00 & 22.21 & 10 & 0.30 & 32.63 & 9 \\
\hline 287 & $12,607.80$ & 4.08 & 14 & 3.76 & $1,880.91$ & 13 & 3.76 & $1,368.72$ & 13 \\
\hline 654 & $237,392.39$ & 12.80 & 12 & 11.59 & $3,521.65$ & 11 & 11.59 & $1,448.62$ & 11 \\
\hline 1060 & $1,874,148.90$ & 41.44 & 15 & 22.90 & $5,420.08$ & 12 & 22.33 & $3,383.88$ & 11 \\
\hline Aver & age & 14.58 & & 9.56 & $2,711.21$ & & 9.49 & $1,558.46$ & \\
\hline
\end{tabular}

For the DLP-CLC problems, according to Table 3 and Table 4, CPLEX was also able to find the optimal solutions when $n=50$ using both the diamond and hexagonal data sets. Similarly to the previous experiments, CPLEX experienced difficulties when the size of the problem becomes relatively large. For all cases, the matheuristic approach yields better average gap compared to the exact method. Moreover, the computational time needed by our matheuristic approach is less than a third of the one required by the exact method. Furthermore, it can also be highlighted that based on \%Gap obtained, the use of Rectilinear distance tends to be harder to solve compared to its counterpart the Euclidean distance. Confirming the previous results, in this case IMA also yielded the smallest \%Gap for Rectilinear case whereas VNS generated better solutions for Euclidean case. In general, VNS runs faster than IMA while yielding good solutions.

\subsection{Continuous problem scenario}

For the continuous problem, the exact method is only implemented for the rectilinear distance case where linearization was taken place. The model with the Euclidean distance on the other hand is a nonlinear problem which cannot be solved using CPLEX. For the CLP-CC and the CLP-CLC with diamond area datasets, due to CPLEX memory issue, the computing time set for CPLEX is 2 hours only to obtain the lower and upper bounds. The results of our matheuristic methods with Euclidean distance are obtained for benchmarking purposes only as no other results are available in the literature.

The computational results for the CLP-CC problem are given in Table 5 while the results of the CLP-CLC problem using the diamond and the hexagonal data sets are presented in Table 6 and Table 7, respectively. For the Euclidean case, \%Gap is calculated based on $Z_{\text {best }}$ instead of lower bound $(L B)$ where $Z_{\text {best }}$ is the 
Table 3 Computational results for the DLP-CLC using diamond area datasets

\begin{tabular}{|c|c|c|c|c|c|c|c|c|c|}
\hline \multirow{2}{*}{$n$} & \multicolumn{3}{|c|}{ Exact Method } & \multicolumn{3}{|c|}{ IMA } & \multicolumn{3}{|c|}{ VNS } \\
\hline & $L B$ & Gap (\%) & $p^{*}$ & Gap (\%) & CPU Time & $p^{*}$ & Gap (\%) & CPU Time & $p^{*}$ \\
\hline \multicolumn{10}{|c|}{ Rectilinear Distance } \\
\hline 50 & 121.94 & 0.00 & 9 & 0.00 & 24.36 & 9 & 0.00 & 22.79 & 9 \\
\hline 287 & $16,935.47$ & 5.06 & 12 & 4.65 & $1,988.75$ & 11 & 4.67 & $1,002.61$ & 11 \\
\hline 654 & $304,886.25$ & 12.28 & 11 & 10.69 & $3,241.89$ & 11 & 10.69 & $1,791.69$ & 11 \\
\hline 1060 & $2,218,010.54$ & 46.59 & 15 & 26.17 & $5,422.06$ & 12 & 27.51 & $3,056.51$ & 12 \\
\hline Aver: & & 15.98 & & 10.38 & $2,669.27$ & & 10.72 & $1,468.40$ & \\
\hline \multicolumn{10}{|c|}{ Euclidean Distance } \\
\hline 50 & 108.53 & 0.00 & 7 & 0.00 & 27.22 & 7 & 0.00 & 27.81 & 7 \\
\hline 287 & $15,808.05$ & 5.03 & 12 & 6.39 & $2,449.80$ & 9 & 4.79 & $1,165.00$ & 11 \\
\hline 654 & $287,842.41$ & 14.70 & 11 & 12.92 & $3,281.38$ & 13 & 12.14 & $1,676.98$ & 11 \\
\hline 1060 & $2,232,653.56$ & 34.65 & 15 & 16.04 & $5,422.96$ & 11 & 17.70 & $2,896.25$ & 11 \\
\hline Avers & & 13.60 & & 8.84 & $2,795.34$ & & 8.66 & $1,441.51$ & \\
\hline
\end{tabular}

Table 4 Computational results for the DLP-CLC using hexagonal area datasets

\begin{tabular}{|c|c|c|c|c|c|c|c|c|c|}
\hline \multirow{2}{*}{$n$} & \multicolumn{3}{|c|}{ Exact Method } & \multicolumn{3}{|c|}{ IMA } & \multicolumn{3}{|c|}{ VNS } \\
\hline & $L B$ & Gap (\%) & $p^{*}$ & Gap (\%) & CPU Time & $p^{*}$ & Gap (\%) & CPU Time & $p^{*}$ \\
\hline \multicolumn{10}{|c|}{ Rectilinear Distance } \\
\hline 50 & 125.75 & 0.00 & 9 & 0.78 & 27.81 & 7 & 0.31 & 35.46 & 10 \\
\hline 287 & $16,937.40$ & 4.35 & 12 & 3.67 & $1,911.47$ & 12 & 3.84 & 807.88 & 11 \\
\hline 654 & $306,557.17$ & 10 & 11 & 06 & $3,140.53$ & 11 & 8.82 & $2,616.83$ & 12 \\
\hline 1060 & $2,440,325.66$ & 39.51 & 15 & 19.62 & $5,421.31$ & 12 & 20.50 & $2,796.30$ & 11 \\
\hline Avera & & 13.24 & & 8.03 & $2,625.28$ & & 8.37 & $1,564.12$ & \\
\hline \multicolumn{10}{|c|}{ Euclidean Distance } \\
\hline 50 & 112.28 & 0.00 & 8 & 0.33 & 22.31 & 7 & 0.33 & 30.22 & 7 \\
\hline 287 & $15,745.06$ & 5.44 & 12 & 4.13 & $2,110.88$ & 11 & 4.14 & $1,238.91$ & 13 \\
\hline 654 & $291,277.77$ & 9.13 & 11 & 9.01 & $3,235.15$ & 10 & 8.75 & $2,100.50$ & 11 \\
\hline 1060 & $2,250,517.00$ & 39.16 & 15 & 18.12 & $5,423.72$ & 11 & 17.67 & $2,908.07$ & 12 \\
\hline Avera & & 13.43 & & 7.90 & $2,698.01$ & & 7.72 & $1,569.42$ & \\
\hline
\end{tabular}

best solution produced by either IMA or VNS. For the continuous problem, the DLP-CLC problem is significantly harder to solve as the location cost of the opening facilities is also a decision variable. The average gap with rectilinear distance in Table 5 and Table 6 confirms this statement where the average \%Gap for the CLP-CLC problem (80.43\%) is much higher than the one for the CLP-CC problem $(60.87 \%)$. According to the computational results shown in Table 5 , it is noted that for the continuous problem the optimal solution cannot be obtained using an exact method (rectilinear case). The \%Gap produced in the continuous problem is also higher than the one obtained in the discrete problem. In line with the previous results, it can also be highlighted here that our matheuristic approaches produces a better (i.e., much lower) average gap than the exact method while requiring a relatively shorter computational time. Here, the performance of IMA and VNS is relatively similar. However, IMA yielded a smaller gap for the Euclidean case. 
Table 5 Computational results for the CLP-CC

\begin{tabular}{|c|c|c|c|c|c|c|c|c|c|c|}
\hline \multirow{2}{*}{$n$} & \multicolumn{3}{|c|}{ Exact Method } & & \multicolumn{3}{|c|}{ IMA } & \multicolumn{3}{|c|}{ VNS } \\
\hline & $L B$ & Gap (\%) & $p^{*}$ & & Gap (\%) & CPU Time & $p^{*}$ & Gap (\%) & CPU Time & $p^{*}$ \\
\hline \multicolumn{11}{|c|}{ Rectilinear Distance } \\
\hline 50 & 45.13 & 59.56 & 10 & & 59.82 & 81.48 & 11 & 59.67 & 48.69 & 10 \\
\hline 287 & $5,372.00$ & 62.02 & 14 & & 62.58 & 879.05 & 13 & 62.67 & 679.53 & 13 \\
\hline 654 & $130,800.00$ & 54.72 & 13 & & 53.62 & $1,219.94$ & 11 & 53.69 & 866.06 & 11 \\
\hline 1060 & $1,060,000.00$ & 67.19 & 15 & & 60.66 & $2,795.36$ & 12 & 60.64 & 961.76 & 12 \\
\hline Avera & age & 60.87 & & & 59.17 & $1,243.96$ & & 59.17 & 639.01 & \\
\hline \multicolumn{11}{|c|}{ Euclidean Distance } \\
\hline 50 & NP & NP & NP & $\begin{array}{c}Z_{\text {best }} \\
100.31\end{array}$ & 0.01 & 216.64 & 9 & 0.00 & 61.10 & 9 \\
\hline 287 & NP & NP & NP & $13,089.03$ & 0.00 & $1,335.45$ & 13 & 0.02 & $1,170.44$ & 12 \\
\hline 654 & NP & NP & NP & $267,993.55$ & 0.00 & $3,716.36$ & 11 & 0.00 & 902.28 & 11 \\
\hline 1060 & NP & NP & NP & $2,402,452.54$ & 0.00 & $7,026.66$ & 11 & 0.17 & $1,618.56$ & 12 \\
\hline Avera & age & & & & 0.00 & $3,073.78$ & & 0.05 & 938.09 & \\
\hline
\end{tabular}

NP: CPLEX cannot be used to solve the Euclidean case as it is a nonlinear problem

\section{Diamond area datasets}

Table 6 shows the computational results for the CLP-CLC problem with diamond area datasets. It can be noted that the matheuristic approach performs also well in tackling this problem as it obtained a smaller average gap compared to the exact method while consuming less computational time. Based on these results, the number of open facilities needed for all instances is less than or equal to 12 whereas the current UB found by the exact method required facilities in the largest instances.

Table 6 Computational results for the CLP-CLC using diamond area datasets

\begin{tabular}{|c|c|c|c|c|c|c|c|c|c|c|}
\hline \multirow{2}{*}{$n$} & \multicolumn{3}{|c|}{ Exact Method } & & \multicolumn{3}{|c|}{ IMA } & \multicolumn{3}{|c|}{ VNS } \\
\hline & $L B$ & Gap (\%) & $p^{*}$ & & Gap (\%) & CPU Time & $p^{*}$ & Gap (\%) & CPU Time & $p^{*}$ \\
\hline \multicolumn{11}{|c|}{ Rectilinear Distance } \\
\hline 50 & 52.84 & 66.94 & 6 & & 56.37 & 70.04 & 9 & 56.46 & 38.22 & 9 \\
\hline 287 & $8,574.65$ & 73.61 & 14 & & 52.11 & 831.17 & 11 & 51.91 & 741.07 & 11 \\
\hline 654 & $130,800.00$ & 90.11 & 15 & & 61.76 & $1,150.19$ & 11 & 61.81 & 933.44 & 11 \\
\hline 1060 & $1,060,946.20$ & 91.06 & 15 & & 66.00 & 548.32 & 11 & 65.31 & 372.35 & 12 \\
\hline Avera & age & 80.43 & & & 59.06 & 649.93 & & 58.87 & 521.27 & \\
\hline \multicolumn{11}{|c|}{ Euclidean Distance } \\
\hline 50 & NP & NP & NP & $\begin{array}{c}Z_{\text {best }} \\
107.77\end{array}$ & 0.03 & 91.49 & 7 & 0.00 & 27.03 & 7 \\
\hline 287 & NP & NP & NP & $16,495.34$ & 0.17 & $1,355.73$ & 12 & 0.00 & $1,211.57$ & 12 \\
\hline 654 & NP & NP & NP & $327,993.55$ & 0.00 & $1,393.19$ & 11 & 0.00 & 609.00 & 11 \\
\hline 1060 & NP & NP & NP & $2,736,352.42$ & 1.18 & 851.63 & 12 & 0.00 & 283.01 & 11 \\
\hline Avera & age & & & & 0.34 & 923.01 & & 0.00 & 532.65 & \\
\hline
\end{tabular}




\section{Hexagonal area datasets}

The computational results for the CLP-CLC problem with hexagonal area datasets are given in Table 7 . In this scenario, the exact method using CPLEX was not able to obtain lower and upper bounds within 24 hours. In line with the experiments on diamond area dataset, VNS outperformed IMA for both Rectilinear and Euclidean cases.

Table 7 Computational results for the CLP-CLC using hexagonal area datasets

\begin{tabular}{|c|c|c|c|c|c|c|c|c|c|c|}
\hline \multirow{2}{*}{$n$} & \multicolumn{3}{|c|}{ Exact Method } & \multirow{2}{*}{$Z_{\text {best }}$} & \multicolumn{3}{|c|}{ IMA } & \multicolumn{3}{|c|}{ VNS } \\
\hline & $L B$ & Gap (\%) & $p^{*}$ & & Gap (\%) & CPU Time & $p^{*}$ & Gap (\%) & CPU Time & $p^{*}$ \\
\hline \multicolumn{11}{|c|}{ Rectilinear Distance } \\
\hline 50 & $\mathrm{NF}$ & NF & NF & 126.27 & 0.67 & 64.21 & 8 & 0.00 & 39.30 & 10 \\
\hline 287 & $\mathrm{NF}$ & $\mathrm{NF}$ & $\mathrm{NF}$ & $17,706.04$ & 0.70 & 781.29 & 13 & 0.00 & 804.54 & 11 \\
\hline 654 & NF & NF & NF & $345,589.32$ & 0.84 & $1,108.11$ & 11 & 0.00 & 355.50 & 12 \\
\hline 1060 & $\mathrm{NF}$ & NF & NF & $3,059,632.21$ & 0.37 & 527.50 & 12 & 0.00 & 384.98 & 12 \\
\hline Avera & age & & & & 0.65 & 620.28 & & 0.00 & 396.08 & \\
\hline \multicolumn{11}{|c|}{ Euclidean Distance } \\
\hline 50 & NP & NP & NP & 112.63 & 0.13 & 171.96 & 7 & 0.00 & 38.82 & 7 \\
\hline 287 & NP & NP & NP & $16,404.82$ & 0.00 & $1,261.00$ & 13 & 0.02 & $1,120.61$ & 11 \\
\hline 654 & NP & NP & NP & $329,742.09$ & 1.28 & 708.37 & 11 & 0.00 & 420.84 & 11 \\
\hline 1060 & NP & NP & NP & $2,773,752.46$ & 0.00 & 646.81 & 11 & 0.54 & 365.18 & 10 \\
\hline Avera & age & & & & 0.35 & 697.03 & & 0.14 & 486.36 & \\
\hline
\end{tabular}

\section{Conclusions}

The single source location problem with the presence of several possible capacities and the opening facility fixed cost that is capacity and zone dependent is investigated. Mathematical models for the discrete and the continuous cases using the Rectilinear and Euclidean distances are produced. As the location models with opening cost for discrete and continuous cases are very hard to solve optimally especially when the size of the problem is large, two matheuristic approaches, which embeds the exact method to tackle the assignment sub problem is put forward, namely an iterative matheuristic approach (IMA) and VNS-based matheuristic. The exact method is first applied where optimal solutions or lower and upper bounds can be found. In our experiments, it can be seen that the matheuristic approaches (IMA and VNS) produce a better average gap than the exact method besides requiring a shorter computational time. For instance, the proposed matheuristics for the discrete problem generate an average \% Gap with just below $9 \%$ compared to around $14 \%$ for the exact method for both rectilinear and Euclidean cases. For the continuous case, these \%Gap averages increased to around $60 \%$ and $70 \%$ for the matheuristics and the exact method respectively, demonstrating the complexity of solving the continuous problem. In general, VNS performs the best as it runs relatively fast while producing good solutions. Note that these \%Gaps may not be as informative as one would like given the lower 
bounds obtained may be too loose. To overcome this issue, one can tighten the formulations by creating new cuts, and also produce tighter upper bounds through more powerful metaheuristics. For future research, it could be interesting to explore other variants including the uncertainty in the customers demand and the presence of forbidden regions. Another exciting aspect that could be pursued is to integrate the routing distances instead of the classical radial ones. This falls into the class of continuous location routing problem (Salhi and Nagy, 2009) which is more challenging but could be worth the investigation.

\section{References}

Manzour-al Ajdad SMH, Torabi SA, Eshghi K (2012) Single-source capacitated multi-facility weber problem - an iterative two phase heuristic algorithm. Computers and Operations Research 39:1465-1476

Akyüs MH, Altnel IK, Öncan T (2014) Location and allocation based branch and bound algorithms for the capacitated multi-facility weber problem. Annals of Operations Research 222(1):45-71

Aras N, Altnel IK, Orbay M (2007a) New heuristic methods for the capacitated multi-facility weber problem. Naval Research Logistics 54:21-32

Aras N, Yumusak S, Altnel IK (2007b) Solving the capacitated multi-facility weber problem by simulated annealing, threshold accepting and genetic algorithms. In: Doerner KF, Gendreau M, P G, Gutjahr WJ, Hartl RF, Reimann M (eds) Metaheuristics: Progress in Complex Systems Optimization, Springer, pp 91-112

Aras N, Orbay M, Altnel IK (2008) Efficient heuristics for the rectilinear distance capacitated multi-facility weber problem. Journal of the Operational Research Society 59:64-79

Arostegui Jr MA, Kadipasaoglu SN, Khumawala BM (2008) An empirical comparison of tabu search, simulated annealing, and genetic algorithms for facilities location problems. Int J Production Economics 103:742-754

Aydin N, Murat A (2013) A swarm intelligence based sample average approximation algorithm for the capacitated reliable facility location problem. Int $\mathrm{J}$ Production Economics 145:173-183

Brimberg J, Mladenović N (1996) A variable neighbourhood algorithm for solving the continuous location-allocation problem. Studies of Locational Analysis 10:112

Brimberg J, Salhi S (2005) A continuous location-allocation problem with zonedependent fixed cost. Annals of Operations Research 136:99-115

Brimberg J, Hansen P, Mladenović N, Taillard ED (2000) Improvements and comparison of heuristics for solving the uncapacitated multisource weber problem. Operations Research 48:444-460

Brimberg J, Hansen P, Mladenović N, Salhi S (2008) A survey of solution methods for the continuous location-allocation problem. International Journal of Operations Research 5:1-12

Brimberg J, Drezner Z, Mladenović N, Salhi S (2014) A new local search for continuous location problems. European Journal of Operational Research 232:256-265

Chen CH, Ting CJ (2008) Combining lagrangian heuristic and ant colony system to solve the single source capacitated facility location problem. Transportation Research Part E: Logistics and Transportation Review 44(6):1099-1122 
Chernov N, Stoyan Y, Romanova T (2009) Mathematical model and efficient algorithms for objects packing problem. Computational Geometry: Theory and Applications 5, DOI 10.1016/ j.comgeo.2009.12.003

Contreras A I, Díaz JA (2008) Scatter search for the single source capacitated facility location problem. Annals of Operations Research 157(1):73-89

Cooper L (1964) Heuristic methods for location-allocation problems. SIAM Review $6: 37-53$

Cooper L (1972) The transportation-location problem. Operations Research 20:94108

Correia I, Captivo ME (2003) A lagrangean heuristic for a modular capacitated location problem. Annals of Operations Research 122:141-161

Correia I, Gouveia L, Saldanha-da Gama F (2010a) Discretized formulations for capacitated location problems with modular distribution costs. European Journal of Operational Research 204:237-244

Correia I, Nickel S, Saldanha-da Gama F (2010b) Single-assignment hub location problems with multiple capacity levels. Transportation Research Part B 44:1047-1066

Daskin MS (2008) What you should know about location modelling. Naval Research Logistics 55:283-294

Drezner T, Drezner Z, Salhi S (2006) A multi objective heuristic approach for the casualty collection points location problem. Journal of the Operational Research Society 57:727-734

Drezner Z, Hamacher H (2002) Facility location: Applications and Theory. Springer, New York

Eiselt HA, Marianov V (eds) (2011) Foundations of Location Analysis. 155, Springer, New York

Farahani RZ, Masoud Hekmatfar M, Fahimnia B, Kazemzadeh N (2014) Hierarchical facility location problem: Models, classifications, techniques, and applications. Computers and Industrial Engineering 68:104-117

Gadegaard SL, Klose A, Nielsen LR (2016) A bi-objective approach to discrete cost-bottleneck location problems. Annals of Operations Research 55, DOI $10.1007 /$ s10479-016-2360-8

Gamal MDH, Salhi S (2001) Constructive heuristics for the uncapacitated locationallocation problem. Journal of the Operational Research Society 51:1233-1240

Gamal MDH, Salhi S (2003) A cellular heuristic for the multisource weber problem. Computers and Operations Research 30:1609-1624

Gong D, Gen M, Yamazaki G, Xu W (1997) Hybrid evolutionary method for capacitated location-allocation problem. Computers and Industrial Engineering $33: 577-580$

Guastaroba G, Speranza MG (2014) A heuristic for bilp problems: The single source capacitated facility location problem. European Journal of Operational Research 238:438-450

Hale T, Moberg C (2003) Location science research: A review. Annals of Operations Research 123:21-35

Hansen P, Mladenović N (1997) Variable neighbourhood search for the $p$-median. Location Science 5(4):207-225

Hansen P, Mladenović N (2001) Variable neighbourhood search: Principles and applications. European Journal of Operational Research 130(3):449-467 
Hansen P, Mladenović N, Taillard ED (1998) Heuristic of the multisource weber problem as a p-median problem. Operation Research Letters 22:55-62

Hansen P, Mladenović N, Perez J (2010) Variable neighbourhood search: methods and applications. Annals of Operations Research 175(1):367-407

Ho SC (2015) An iterated tabu search heuristic for the single source capacitated facility location problem. Applied Soft Computing 27:169-178

Hosseininezhad SJ, Salhi S, Jabalameli MS (2015) A cross entropy-based heuristic for the capacitated multi-source weber problem with facility fixed cost. Computers and Industrial Engineering 83:151-158

Irawan CA, Salhi S, Luis M, Azizi N (2017) The continuous single source location problem with capacity and zone-dependent fixed cost: Models and solution approaches. European Journal of Operational Research 263:94-107

Klose A, Drexl A (2005) Facility location models for distribution system design. European Journal of Operational Research 162:4-29

Lin CKY (2009) Stochastic single-source capacitated facility location model with service level requirements. Int J Production Economics 117:439-451

Luis M, Salhi S, Nagy G (2009) Region-rejection based heuristics for the capacitated multi-source weber problem. Computers and Operations Research 36:2007-2017

Luis M, Salhi S, Nagy G (2011) A guided reactive grasp for the capacitated multisource weber problem. Computers and Operations Research 38:1014-1024

Luis M, Salhi S, Nagy G (2015) A constructive method and a guided hybrid grasp for the capacitated multi-source weber problem in the presence of fixed cost. Journal of Algorithms and Computational Technology 9:215-232

Manzour H, Torabi A, Pishvaee MS (2013) New heuristic methods for the singlesource capacitated multi facility weber problem. International Journal of Advanced Manufacturing Technology 69:1569-1579

Megiddo N, Supowit KJ (1984) On the complexity of some common geometric location problems. SIAM Journal on Computing 13:182-196

Mohammadi N, Malek MR, Alesheikh AA (2010) A new ga based solution for capacitated multi source weber problem. International Journal of Computational Intelligence Systems 3:514-521

Öncan T (2013) Heuristics for the single source capacitated multi-facility weber problem. Computers and Industrial Engineering 64:959-971

Rahmani A, MirHassani SA (2014) A hybrid firefly-genetic algorithm for the capacitated facility location problem. Information Sciences 283:70-78

Salhi S (2017) Heuristic Search: The Emerging Science of Problem Solving. Springer, Switzerland

Salhi S, Nagy G (2009) Local improvement in planar facility location using vehicle routing. Annals of Operations Research 167:287-296

Sherali HD, Nordai FL (1988) Np-hard, capacitated, balanced p-median problems on a chain graph with a continuum of link demands. Mathematics of Operations Research 13:32-49

Yang Z, Chu F, Chen H (2012) A cut-and-solve based algorithm for the single source capacitated facility location problem. European Journal of Operational Research 221(3):521-532

Zainuddin ZM, Salhi S (2007) A perturbation-based heuristic for the capacitated multisource weber problem. European Journal of Operational Research 179:1194-1207 
Hansen P, Mladenović N, Taillard ED (1998) Heuristic of the multisource weber problem as a p-median problem. Operation Research Letters 22:55-62

Hansen P, Mladenović N, Perez J (2010) Variable neighbourhood search: methods and applications. Annals of Operations Research 175(1):367-407

Ho SC (2015) An iterated tabu search heuristic for the single source capacitated facility location problem. Applied Soft Computing 27:169-178

Hosseininezhad SJ, Salhi S, Jabalameli MS (2015) A cross entropy-based heuristic for the capacitated multi-source weber problem with facility fixed cost. Computers and Industrial Engineering 83:151-158

Irawan CA, Salhi S, Luis M, Azizi N (2017) The continuous single source location problem with capacity and zone-dependent fixed cost: Models and solution approaches. European Journal of Operational Research 263:94-107

Klose A, Drexl A (2005) Facility location models for distribution system design. European Journal of Operational Research 162:4-29

Lin CKY (2009) Stochastic single-source capacitated facility location model with service level requirements. Int J Production Economics 117:439-451

Luis M, Salhi S, Nagy G (2009) Region-rejection based heuristics for the capacitated multi-source weber problem. Computers and Operations Research 36:2007-2017

Luis M, Salhi S, Nagy G (2011) A guided reactive grasp for the capacitated multisource weber problem. Computers and Operations Research 38:1014-1024

Luis M, Salhi S, Nagy G (2015) A constructive method and a guided hybrid grasp for the capacitated multi-source weber problem in the presence of fixed cost. Journal of Algorithms and Computational Technology 9:215-232

Manzour H, Torabi A, Pishvaee MS (2013) New heuristic methods for the singlesource capacitated multi facility weber problem. International Journal of Advanced Manufacturing Technology 69:1569-1579

Megiddo N, Supowit KJ (1984) On the complexity of some common geometric location problems. SIAM Journal on Computing 13:182-196

Mohammadi N, Malek MR, Alesheikh AA (2010) A new ga based solution for capacitated multi source weber problem. International Journal of Computational Intelligence Systems 3:514-521

Öncan T (2013) Heuristics for the single source capacitated multi-facility weber problem. Computers and Industrial Engineering 64:959-971

Rahmani A, MirHassani SA (2014) A hybrid firefly-genetic algorithm for the capacitated facility location problem. Information Sciences 283:70-78

Salhi S (2017) Heuristic Search: The Emerging Science of Problem Solving. Springer, Switzerland

Salhi S, Nagy G (2009) Local improvement in planar facility location using vehicle routing. Annals of Operations Research 167:287-296

Sherali HD, Nordai FL (1988) Np-hard, capacitated, balanced p-median problems on a chain graph with a continuum of link demands. Mathematics of Operations Research 13:32-49

Yang Z, Chu F, Chen H (2012) A cut-and-solve based algorithm for the single source capacitated facility location problem. European Journal of Operational Research 221(3):521-532

Zainuddin ZM, Salhi S (2007) A perturbation-based heuristic for the capacitated multisource weber problem. European Journal of Operational Research 179:1194-1207 